\title{
Ventilatory and Heart Rate Responses at the Onset of Chair Rotation in Man
}

\author{
Miharu MIYAMURA, Koji ISHIDA*, Keisho KATAYAMA*, Norihiro SHIMA ${ }^{\dagger}$, \\ Hiroshi MATSUO*, and Kohei SATO*
}

\begin{abstract}
The Faculty of Wellness, Tokai Gakuen University, Nagoya, 468-8514 Japan; *Research Center of Health, Physical Fitness and Sports, Nagoya University, Nagoya, 464-8601 Japan; and ${ }^{\dagger}$ National Institute of Fitness and Sports, Kanoya, 891-2393 Japan
\end{abstract}

\begin{abstract}
In the present study, we attempted to confirm whether pulmonary ventilation and heart rate increased immediately after passive chair rotation in man. Inspiratory minute volume $\left(\dot{V}_{\mathrm{I}}\right)$, tidal volume $\left(V_{T}\right)$, respiratory frequency $(f)$, and heart rate $(\mathrm{HR})$ were determined by breath-bybreath and beat-by-beat techniques before, during, and after rotation for a total of $45 \mathrm{~s}$. It was found that $\dot{V}$ s significantly increased immediate-
\end{abstract}

ly after chair rotation, but HR remained almost constant. These results suggest that the activation of horizontal semicircular canals is one causal factor of ventilatory response at the onset of exercise with rotational movement in healthy subjects, but heart rate response is not. [The Japanese Journal of Physiology 54: 499-503, 2004]

Key words: phase I, semicircular canal, human.

$\mathrm{I}_{\mathrm{t}}$ rate both increase immediately after light or moderate exercise. This abrupt increase in ventilation has been shown to occurs usually from the first breath [1] and is termed phase I [2]. This phase I response, lasting about $15 \mathrm{~s}$, is observed not only during voluntary exercise, but also during passive movement following electrically induced muscle contractions or flexion extensions of the lower legs with ropes $[3,4]$.

Nevertheless, many investigators have pursued mechanisms that are responsible for the phase I response, and their opinions about its nature are still a matter of dispute. At present, it is considered that two neural mechanisms, central command and peripheral reflex, mainly trigger the increase in ventilation that appears at the onset of exercise [5-7]. Central command arises from the activation of the cerebral cortex and hypothalamus [8,9], and peripheral reflex originates in the stimulation of groups III and IV muscle afferents $[10,11]$. Recently, a stimulation of the vestibular system has been reported to elicit cardiovascular and respiratory changes $[12,13]$. In support of this concept, Jauregui-Renaud et al. [14] observed that a stimulation of the vertical semicircular canal increased breathing frequency in normal subjects, but not in vestibular-deficient patients. Monahan et al. [15] also reported significant changes in respiratory frequency and minute ventilation from the baseline during dynamic chair rotation for one minute. From these results, it is possible to hypothesize that an abrupt increase in minute ventilation and heart rate immediately after exercise may be related not only to central command and afferent impulses from working muscles through groups III and IV, but also to semicircular canals that would be stimulated in various sports situations, such as rotation in ice skating or artistic gymnastics. To our knowledge, however, no data are available concerning this hypothesis in healthy subjects. The purpose of this study, therefore, was to clarify whether the horizontal semicircular canal afferents mediate the ventilatory and heart rate responses at the onset of passive chair rotation in man.

\section{METHODS}

The present study was approved by the Human Research Committee of the Research Center of

Received on July 1, 2004; accepted on October 4, 2004

Correspondence should be addressed to: Miharu Miyamura, The Faculty of Wellness, Tokai Gakuen University, Nagoya, $468-8514$ Japan. Tel: +81-52-801-6979, Fax: +81-52-804-1044, E-mail: miyamura@tokaigakuen-c.ac.jp 
Health, Physical Fitness and Sports of Nagoya University. Eight healthy men volunteered to participate, and they were informed of the experimental protocol and possible risks involved in the study before giving written consent. None had a history of cardiorespiratory disease, of medications that seriously affected cardiorespiratory response, or of tabaco. The mean and standard errors of age, height, and weight of the subjects were 27.0 (1.92) years, 171.6 $(1.20) \mathrm{cm}$, and $63.1(2.40) \mathrm{kg}$.

The vestibular stimulus tests were carried out on each subject by using chair rotations; i.e., during the experiment, the subject sat with his back against an experimental swivel chair. Each subject's torso and head were securely fastened to the chair with belts. The chair could be swiveled $360^{\circ}$ about a central axis by an experimenter, and a rotation would be denoted as either "right turn" or "left turn." After resting comfortably in a sitting position for $20 \mathrm{~min}$, the subject was asked to relax during experiment. The rotation chair was turned $180^{\circ}$ manually by an experimenter for $1.5(1.4-1.6) \mathrm{s}$ in mean from right to left or the reverse.

The right-turn and the left-turn were repeated randomly three times at intervals of approximately a few minutes; the subject's eyes were closed to exclude the effects of the field of vision. These rotating tests were initiated just before the start of the inspiratory phase, and the experimenter observed the activity on an oscilloscope connected to a hot-wire flow meter (RF-H, Minato Ikagaku, Japan). The goniometer was attached to the axis of the turn chair to detect the start of turning. Inspiratory minute volume $\left(\dot{V}_{\mathrm{I}}\right)$, tidal volume $(V \mathrm{~T})$, and inspiratory and expiratory periods were determined by the breath-by-breath technique before, during, and after turning for a total of $45 \mathrm{~s}$. That is, $\dot{V}_{\mathrm{I}}$ and $V$ T were measured continuously for $30 \mathrm{~s}$ before, $1.5 \mathrm{~s}$ during, and $13.5 \mathrm{~s}$ after chair rotation. The subject breathed through a respiratory face mask attached to a hot-wire flow meter. The respiratory frequency $(f)$ was calculated from the total respiratory time, and $\dot{V}$ I was obtained as the product of $V$ T and $f$. Furthermore, heart rate (HR) was also monitored beat-to-beat by using electrocardiography before, during and after rotation for a total of $45 \mathrm{~s}$. All ventilatory and circulatory signals were converted from analog to digital data with an A/D converter (Canopus, ADX 98H, Japan) at a sampling frequency of $100 \mathrm{~Hz}$. These data were stored on a hard disk unit, and analyzed afterward on a personal computer (NEC, PC-9821Xa, Japan). Breath-by-breath and beat-by-beat data were aligned with the onset of each turn, linearly interpolated between each breath or beat to yield a data point at each $1 \mathrm{~s}$ interval, and the ensemble average was done across all three repetitions $[16,17]$. The peak attained times of $\dot{V}_{\mathrm{I}}, V \mathrm{~T}$, and $f$ for each subject were calculated in both rightand left-turn conditions. The changes in absolute values of the parameters during and after a turn, compared with the rest levels, were analyzed in regard to variance with repeated measurements. If a significant $F$ ratio $(p<0.05)$ was obtained, Dunnett's test was used to determine when the differences during the turn occurred. In comparing a left-turn with a rightturn, the Wilcoxon test was used. The level of significance was set at 0.05 .

\section{RESULTS}

Figure 1 shows the results of ventilatory response before, during, and after chair rotation in both the left-turn and the right-turn. $\dot{V}_{\text {I }}$ increased significantly $(p<0.05)$ immediately after rotation. $V \mathrm{~T}$ also increased at the onset of rotation, except at $1 \mathrm{~s}$ in the left-turn. In contrast, f unchanged immediately after rotation in both the left-turns and the right-turns, except at $2 \mathrm{~s}$ in the left turn. Mean values ( \pm SE) of peak attained time of $\dot{V} \mathrm{I}, V \mathrm{~T}$, and $f$ were 3.7 (1.1), 2.6 (0.6), and 5.1 (2.0) s for the right-turn and $2.6(0.2)$, $2.4(0.3)$, and $3.7(1.4) \mathrm{s}$ for the left-turn. There are no significant differences in the peak attained times of $\dot{V}$ I, $V \mathrm{~T}$, and $f$ between the right-turn and the leftturn. And as indicated in Fig. 2, HR did not increase significantly by chair rotation in either condition, except for the value obtained at $2 \mathrm{~s}$ after the beginning of left rotation. Since the values at rest of $\dot{V}$, $V \mathrm{~T}, f$, and HR were different in the two turns, we compared the differences in ventilatory and circulatory responses at the onset of the right-turn and the leftturn by the $\Delta$ value estimated from a mean value of rest for $30 \mathrm{~s}$ of $100 \%$. The $\Delta \dot{V}_{\mathrm{I}}$ and $\Delta V \mathrm{~T}$ increased immediately after rotation in both conditions. The $\Delta \dot{V}_{\mathrm{I}}$ was significantly $(p<0.05)$ greater in the left-turn than in the right-turn at $2-5 \mathrm{~s}$, but no significant difference was found in $\Delta V \mathrm{~T}$. Compared with the right turn, the $\Delta f$ was significantly $(p<0.05)$ greater in the left-turn at $1-3$. On the other hand, no significant difference was observed in $\Delta \mathrm{HR}$ between the right-turn and the left-turn, except at $10 \mathrm{~s}$ in $\Delta \mathrm{HR}$.

\section{DISCUSSION}

In the present study, we attempted to confirm whether pulmonary ventilation increases at the onset of passive chair rotation in healthy subjects. It was found that $\dot{V}$ I significantly increased immediately after chair 

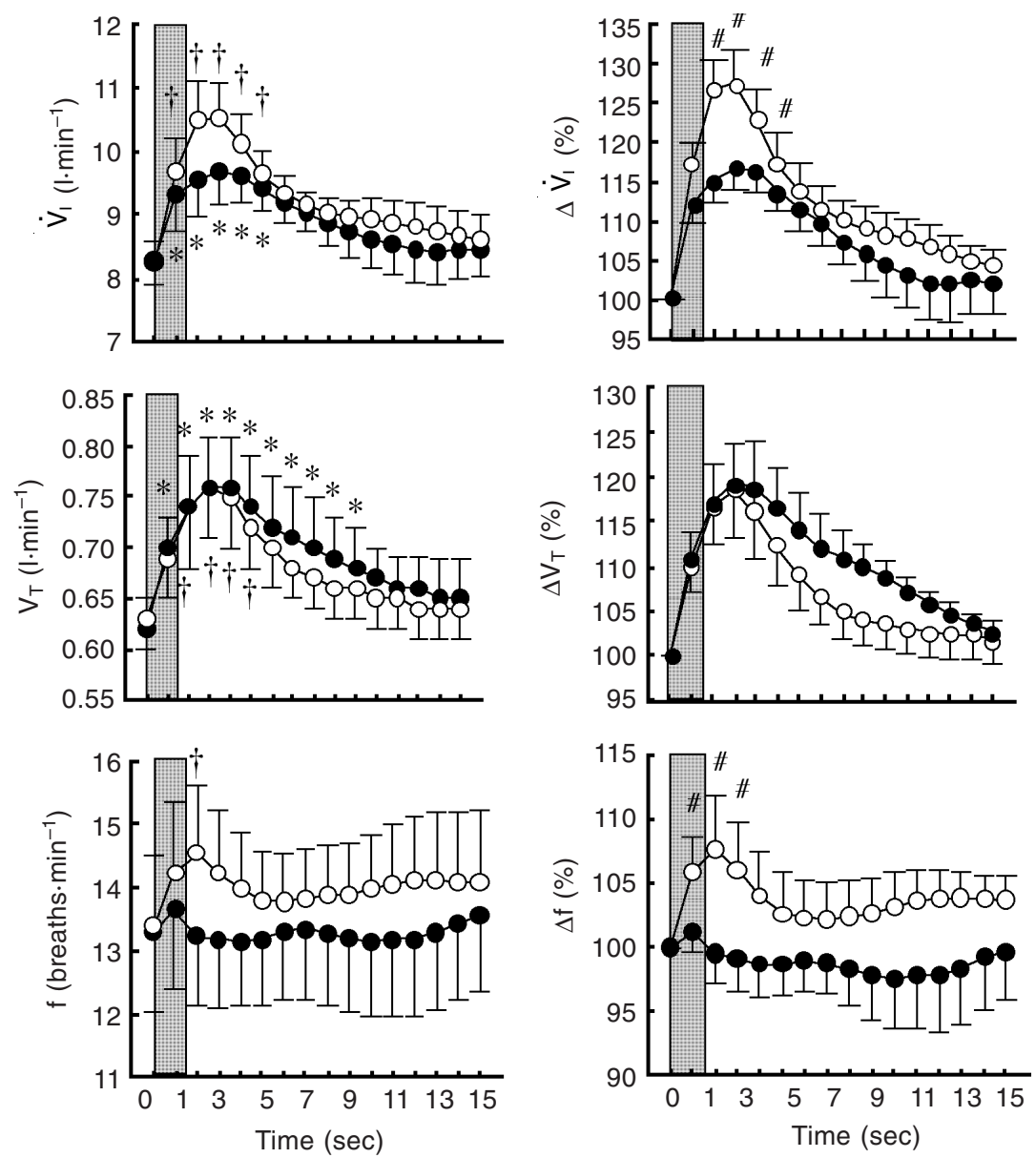

Fig. 1. Absolute changes (left panel) and relative changes (right panel) in inspiratory minute volume ( $\left.\dot{V_{I}}\right)$, tidal volume $(V T)$ and respiratory frequency $(f)$ before, during and after chair rotation in left-turns (opened circles) and right-turns (closed circles). Values in the right panel are absolute values that were converted by interpolation into values for each second. The percentage changes in inspiratory minute volume $(\Delta \dot{V}$ I), tidal volume $(\Delta V T)$ and respiratory frequency $(\Delta f)$ in the right-turn and left-turn when the average of the values at rest was $100 \%$. The turn period is shown

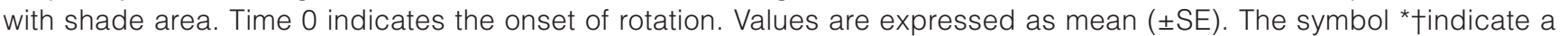
significant difference $(p<0.05)$ from rest, and the symbol \# indicates a significant difference $(p<0.05)$ between the right-turn and the left-turn.
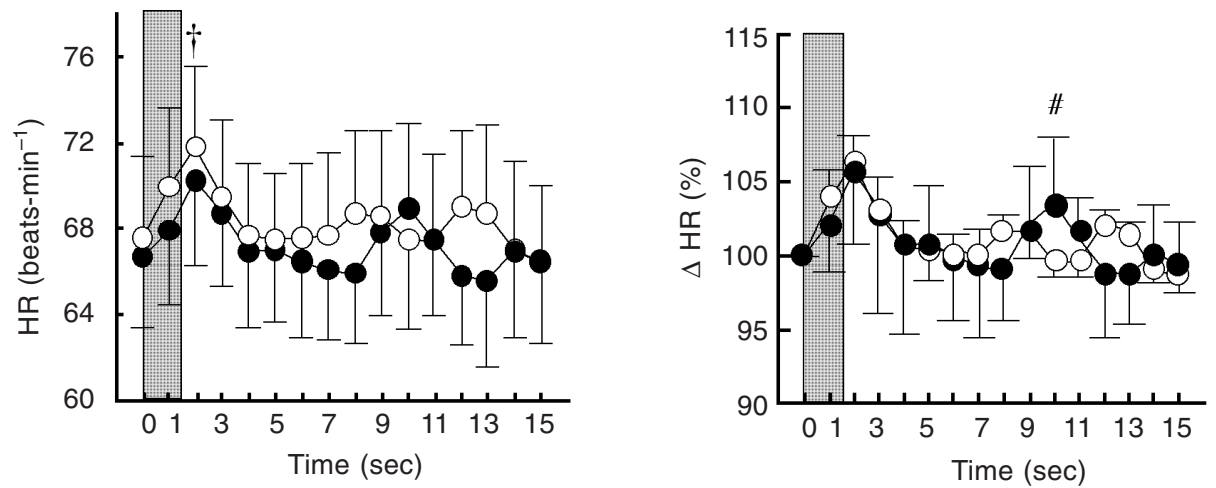

Fig. 2. Absolute changes (left panel) and relative changes (right panel) in heart rate (HR) before, during and after chair rotation in the left-turn (opened circles) and the right-turn (closed circles). The values in the right panel are absolute values, which were converted by interpolation into values for each second. The percentage changes in heart rate $(\Delta \mathrm{HR})$ in the right-turn and left-turn when the average of the values at rest is $100 \%$. The turn period is shown as a shaded area. Time 0 indicates the onset of rotation. The values are expressed as mean ( $\pm S E)$. The symbol $\dagger$ indicates a significant difference $(p<0.05)$ from rest, and the symbol \# indicates a significant difference $(p<0.05)$ between the right and the left-turns. 
rotation in both the right- and left-turns, but not $f$ or HR, as shown in Figs. 1 and 2. We believe this is the first study to show a contribution of the horizontal semicircular canal to ventilatory responses at the onset of passive rotation in humans.

In the previous study, Monahan et al. [15] observed that breathing frequency and minute ventilation were increased, whereas tidal volume is unchanged during chair rotation. In contrast, it was found in the present study that minute ventilation and tidal volume increased immediately after chair rotation, but respiratory frequency did not. The difference in the results obtained by Monahan et al. and those in the present study may be due to the differences in the time, velocity, and maneuver of chair rotation. On the other hand, $\Delta \dot{V}_{\mathrm{I}}$ and $\Delta f$ were significantly $(p<$ $0.05)$ greater in the left-turn compared with the rightturn, as shown in Fig. 1. We cannot now explain why these results were obtained. It may be possible to assume that the right and left differences in the sensitivity of semicircular canals exist even if the subjects are healthy men. However, this assumption requires further investigation.

Since the changes in ventilation are so rapid during the transition from rest to exercise, phase I response cannot be explained by humoral agents because of the delay in transport. At present, the causal factors of phase I are classified largely into central and peripheral neurogenic stimuli, or both $[6$, 9]. Jauregui-Renaud et al. [14] recently measured respiratory frequency during rotational stimuli by using a motorized rotating chair with acceleration in $<1 \mathrm{~s}$ to a constant rotational velocity of $60 \%$ s on vertical axis, sustained for $1 \mathrm{~min}$. They observed that in normal subjects, rotation in the pitch plane elicited an increase in respiratory frequency because of a shortening of the time for expiration. From these results, they conclude that vertical semicircular activity exerts a regulatory drive on respiration in human subjects. Furthermore, Monahan et al. [15] have determined cardiorespiratory parameters such as inspiratory time, expiratory time, ventilation, heart rate, and mean blood pressure during seven various conditions (dynamic upright pitch, dynamic lateral pitch, dynamic head roll, dynamic yaw, dynamic chair rotation, static head down rotation, and static head rotation in the lateral decubitus position) in order to confirm the hypothesis that the activation of the semicircular canals would increase respiration in humans. Since significant changes in inspiratory time, expiratory time, and minute ventilation from the baseline during dynamic chair rotation of $15 \mathrm{cycle} / \mathrm{min}$ for one minute were observed, they suggested that the semicircular canal, but not the otolith organs or neck muscle afferents, mediates increased ventilation in humans and supports the concept that vestibular activation alters respiration in humans. Because they did not indicate the results of respiratory parameters obtained by the breath-by-breath technique for $15 \mathrm{~s}$ from the start of chair rotation, however, it is unclear whether this horizontal vestibular activation produced functional alterations in phase I. In other words, it is important to emphasize that ventilatory and heart rate responses should be determined continuously with breath-by-breath and beat-by-beat techniques within $15 \mathrm{~s}$ rather than one minute total in order to clarify whether a horizontal semicircular canal stimulus is related to ventilatory response (phase I) at the onset of exercise.

As described previously, it is very possible that semicircular canals would be stimulated in various situations of actual physical exercise and sports, such as rotations in ice skating or in artistic gymnastics. In the present study, $\dot{V}$ I increased significantly immediately after passive chair rotation, but HR was unchanged at the onset of chair rotation in both leftand right-turns, as shown in Figs. 1 and 2. We could not compare the ventilatory kinetics with respect to time constant and amplitude between chair rotation and exercise stimuli, since rotation time of the present study was very short $(1.5 \mathrm{~s})$. So, we calculated peak attained time of $\dot{V}$ I for each subject in both leftand right-turn conditions. The mean values of peak attained time of $\dot{V}_{\text {I }}$ were $3.6 \mathrm{~s}$ for the right-turn and $2.6 \mathrm{~s}$ for the left-turn. These results suggest that the activation of the horizontal semicircular canal is one causal factor of ventilatory response in human subjects at the onset of exercise with rotational movement, but not of heart rate response. Although afferent signals from the body trunk and/or neck through groups III and IV could have also made some contribution to the abrupt increase in $\dot{V}_{\mathrm{I}}$ and $V \mathrm{~T}$ immediately after rotation, it is unlikely that the contributions of group III and IV afferents was mainly caused by the increase of $\dot{V}_{\mathrm{I}}$ and $V \mathrm{~T}$ in the present study because the subject's torso and head were securely fastened with belts in a rotation chair. However, we cannot explain based on physiological grounds why respiratory frequency and heart rate are unchanged at the onset of chair rotation in both conditions. It will be necessary to conduct further investigation why $f$ and HR remained constant, though $\dot{V}$ I and $V$ T significantly increased immediately after passive rotation. 


\section{REFERENCES}

1. Eldridge FL, and Waldrop TG: Neural control of breathing during exercise. In : Whipp BJ, Wasserman $\mathrm{K}$ (ed): Exercise : pulmonary physiology and pathology. Marcel Dekker, New York, pp 309-370, 1991

2. Whipp BJ, Ward SA, Lamara N, Davis JA, and Wasserman K: Parameters of ventilatory and gas exchange dynamics during exercise. J Appl Physiol 52: 1506-1513, 1982

3. Adams L, Guz A, Innes JA, and Murphy K: The early circulatory and ventilatory response to voluntary and electrically induced exercise in man. J Physiol (Lond) 383: 19-30, 1987

4. Miyamura M, Ishida K, and Yasuda Y: Ventilatory response to the onset of passive and active exercise in human subjects. Jpn J Physiol 42: 607-615, 1992

5. Mitchell $\mathrm{JH}$ : Neural control of the circulation during exercise. Med Sci Sports Exerc 22: 141-154, 1990

6. Miyamura M: Control of ventilation during exercise in man with special reference to the feature at the onset. Jpn J Physiol 44: 123-139, 1994

7. Mateika JH and Duffin J: A review of the control of breathing during exercise. Eur J Appl Physiol 71: 1-27, 1995

8. Goodwin GM, McCloskey DI, and Mitchell JH: Cardiovascular and respiratory responses to changes in central command during isometric exercise at constant muscle tension. J Physiol (Lond) 226: 173-190, 1972

9. Williamson JW, McColl R, and Mathews D: Evidence for central command activation of the human insular cortex during exercise. J Appl Physiol 94: 1726-1734 2003

10. McCloskey DI, and Mitchell JH: Reflex cardiovascular and respiratory responses originating in exercising muscle. J Physiol (Lond) 224: 173-186, 1972

11. Kaufman MP, Longhurst JC, Rybicki KJ, Wallach JH and Mitchell JH: Effects of static muscular contraction on impulse activity of groups III and IV afferents in cats. J Appl Physiol 55: 105-112, 1983

12. Yates BJ and Miller AD: Physiological evidence that the vestibular system participates in autonomic and respiratory control. J Vestib Res 8: 17-25, 1998

13. Biaggioni I, Costa $F$, and Kaufmann $\mathrm{H}$ : Vestibular influences on autonomic cardiovascular control in humans. J Vestib Res 8: 35-41, 1998

14. Jauregui-Renaud K, Gresty MA, Reynolds R, and Bronstein AM: Respiratory responses of normal and vestibular defective human subjects to rotation in the yaw and pitch planes. Neurosci Lett 298: 17-20, 2001

15. Monahan K, Sharpe MK, Drury D, Ertl AC, and Ray CA: Influence of vestibular activation on respiration in humans. Am J Physiol 282: R689-R694, 2002

16. Miyamura M, Ishida K, Hashimoto I, and Yuza N: Ventilatory response at the onset of voluntary exercise and passive movement in endurance runners. Eur J Appl Physiol 76: 221-229, 1997

17. Ishida K, Sato Y, Katayama K, and Miyamura M: Initial ventilatory and circulatory responses to dynamic exercise are slowed in the elderly. J Appl Physiol 89: $1771-1777,2000$ 